\title{
A Computational Investigation of Unsteady Aerodynamics of Insect-Inspired Fixed Wing Micro Aerial Vehicle's 2D Airfoil
}

\author{
Somashekar V \\ Department of Aeronautical Engineering, Visvesvaraya Technological University, Belgaum, Karnataka 590018, India \\ Correspondence should be addressed to Somashekar V; shekar.mtech.ph.d@gmail.com
}

Received 24 January 2014; Revised 16 May 2014; Accepted 19 May 2014; Published 17 June 2014

Academic Editor: Hamid M. Lankarani

Copyright (C) 2014 Somashekar V. This is an open access article distributed under the Creative Commons Attribution License, which permits unrestricted use, distribution, and reproduction in any medium, provided the original work is properly cited.

\begin{abstract}
A Micro air vehicle (MAV) is defined as class of unmanned air vehicle (UAV) having a linear dimension of less than 15 centimeters and a mass of less than 100 grams with flight speeds of 6 to 12 meters per second. MAVs fall within a Reynolds number (Re) range of 50,000 and 120,000, in which many causes of unsteady aerodynamic effects are not fully understood. The research field of low Reynolds number aerodynamics is currently an active one, with many defence organizations, universities, and corporations working towards a better understanding of the physical processes of this aerodynamic regime. In the present work, it is proposed to study the unsteady aerodynamic analysis of 2D airfoil using CFD software and Xfoil panel code method. The various steps involved in this work are geometric modelling using CATIA V5R17, meshing using ICEM CFD, and solution and postprocessing through FLUENT. The finite control volume analysis and Xfoil panel code method has been carried out to predict aerodynamic characteristics such as lift coefficients, drag coefficients, moment coefficients, pressure coefficients, and flow visualization. The lift and drag coefficients were compared for all the simulations with experimental results. It was observed that for the 2D airfoil, lift and drag both compared well for the midrange angle of attack from -10 to 15 degree AOA.
\end{abstract}

\section{Introduction}

Micro air vehicles (MAVs) have attracted significant attention since mid-1990 for both civilian and military applications. Micro air vehicle (MAV) is defined here as a small, portable flying vehicle which is designed for performing useful work. The desire for portable, low altitude aerial surveillance has driven the development of aircraft on the scale of small birds. Vehicles in this class of small-scale aircraft are known as micro air vehicles or MAVs and have great potential for applications in surveillance and monitoring tasks in areas either too remote or too dangerous to send human agents. Equipped with small video cameras and transmitters, MAVs can image targets that would otherwise remain inaccessible. MAVs are also capable of carrying an array of sensors to obtain additional information including, for example, airborne chemical or radiation levels.

MAVs are by definition small aircrafts which fly at relatively low speeds. Such flight characteristics will result in flow regimes with Reynolds numbers. Another aerodynamic signature of MAV is wings with small aspect ratio; in most cases the chord is roughly equal to the wingspan. This combination of low Reynolds number flight and low aspect ratio wings results in a flow regime totally alien to conventional aircraft. Although small birds and insects have been flying under these conditions for quite some time (Figure 1), this is a new flight environment for man-made aircraft.

In order for required MAV capabilities to be realized, several areas will need more focused attention. The absence of sophisticated computational analysis methods, lack of commercially available micro electromechanical sensors, and the difficulties associated with accurate experimental work at this scale have all restricted research. From a system and manufacturing standpoint, technological advances in micro fabrication techniques and in the miniaturization of electronics in the last decade made mechanical MAVs feasible. Key research challenges include unsteady aerodynamics at low Reynolds number, low aspect ratio wings, stability and control issues associated with low weight, small moments of inertia, miniaturization, cooperative control, and micro sensors. Among these areas unsteady aerodynamics is an important area of research. 


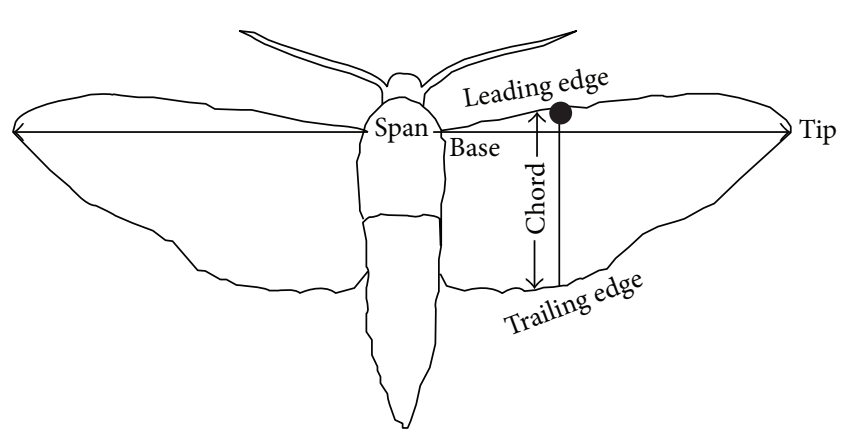

FIgURE 1: Conventions and terminology. Sketch of an insect [1].

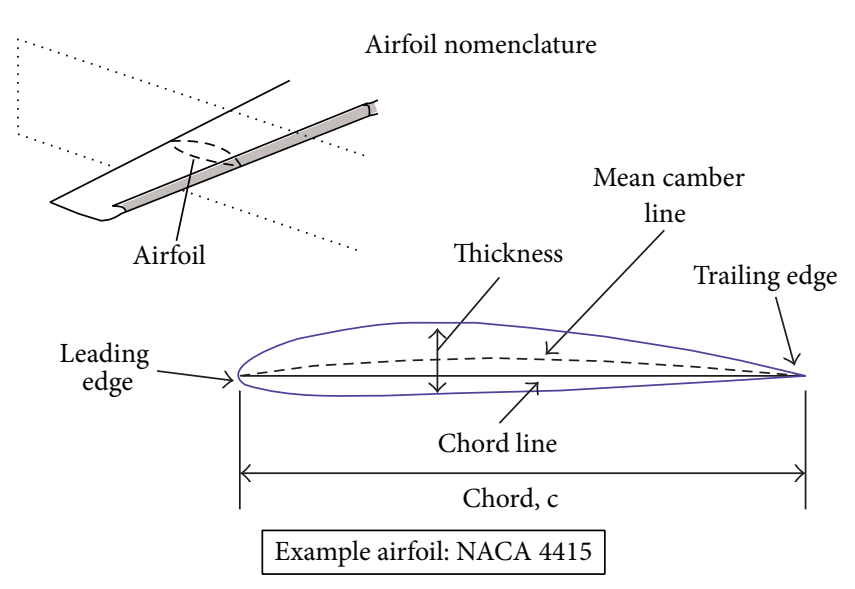

FIGURE 2: Airfoil nomenclatures.

Unsteady aerodynamics plays a significant role in MAV flight and stability. Unsteady phenomena may arise due to natural time dependent changes in the flow itself or it may be created by changes in the position or orientation of a body. In many unsteady flows of interest, the important unsteady aspects involve not only the kinematic changes in boundary conditions caused by the motion of a body but also the influence of an unsteady wake and the changes in the pressure-velocity relationship associated with the unsteady form of Bernoulli's equation [2].

Below is given a $2 \mathrm{D}$ linear translation of a wing of an airfoil (Figure 2).

A 2D linear translation, (Figure 3) as an airfoil begins motion from rest, it generates a leading and trailing edge vortex. During translation, the trailing edge vortex is shed, leading to the growth of the leading edge vortex, which also sheds as the airfoil continues to translate. This motion leads to an alternate vortex shedding pattern from the leading and trailing edges, called the Von Karman vortex street. This leads to a time dependence of the net aerodynamic forces (blue arrows) measured on the airfoil [1].

Mechanisms such as rotational circulation, wake capture, and the unsteady leading edge vortex do seem to properly account for the aerodynamics forces. Regarding forward flight, the unsteady leading edge vortex is the only mechanism present to produce the necessary forces. The unsteady leading edge vortex involves leading edge flow separation that reattaches to the wing and forms a separation bubble.

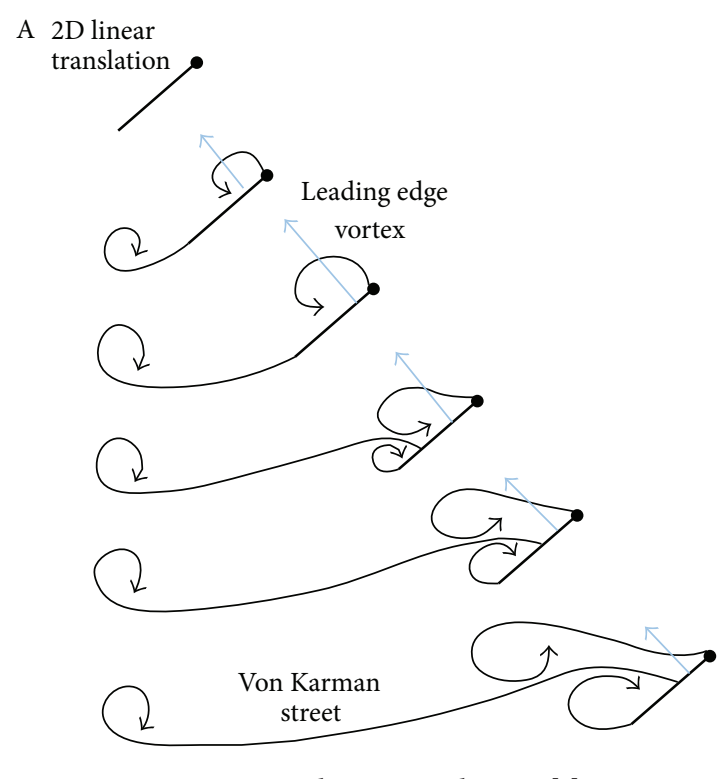

Figure 3: 2D linear translations [1].

The vortex increases the circulation around the wing and creates much higher lift than the steady-state case. This vortex remains stable due to its highly three-dimensional nature.

Low Reynolds numbers make the problem of airfoil design difficult because the boundary layer is much less capable of handling an adverse pressure gradient without separation. Thus, very low Reynolds number designs do not have severe pressure gradients and the maximum lift capability is restricted. At very low Reynolds numbers, most or all of the boundary layer is laminar. Laminar separation bubbles are common and unless properly stabilized can lead to excessive drag and low maximum lift [3].

\section{Methodology}

The focus of the proposed study is to determine the characteristics of unsteady aerodynamics of 2D airfoil at different angle of attack during operation and to achieve at an optimum result against the experimental and existing literature results. The rigorous work involves the modeling and analysis of the entire fixed wing MAV 2D airfoil using CATIA, ANSYS FLUENT, and ICEM-CFD. Various angle of attack 2D airfoil characteristics such as pressure distribution, lift coefficient, drag coefficient, and moment coefficient over the fixed wing MAV 2D airfoil at different flight conditions will be studied and an optimum configuration will be suggested for safe operations. The following governing equations are the prime drivers for the computation of the aerodynamic characteristics.

The flow chart in Figure 4 represents the step by step working procedure of this work. Using Xfoil panel code method predicts the aerodynamic characteristics. The right block shows the standard procedure for CFD; the standard procedure for ICEM-CFD work follows the above components. For the present work, in case of $2 \mathrm{D}$ importing the geometry then computational domain is constructed around 


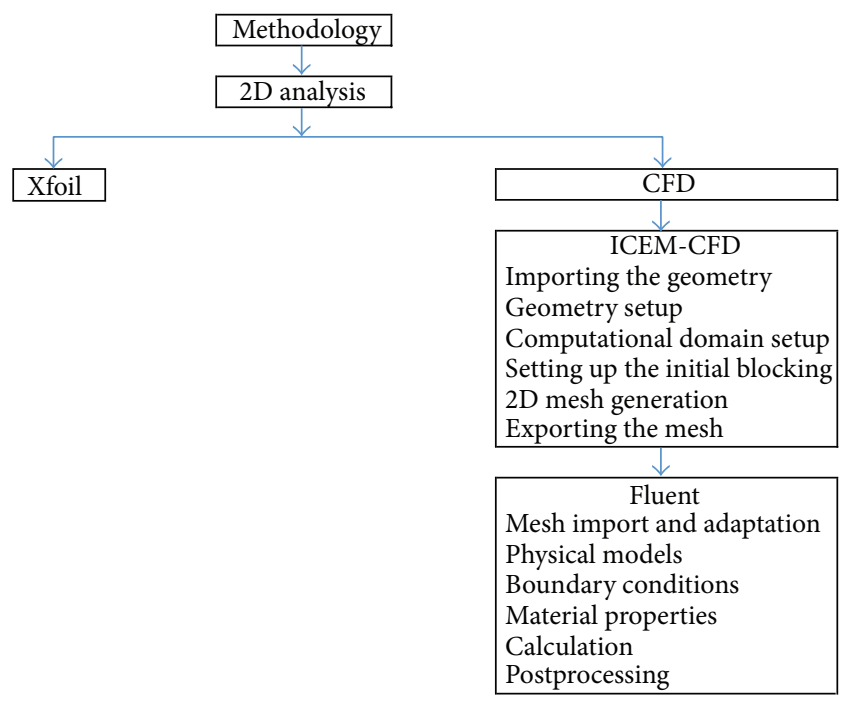

FIgURE 4: Analysis procedure and the components.

the airfoil geometry then setting up the initial blocking. Later set the mesh parameters then generates the mesh. Later this mesh is exported in the fluent readable format.

The standard procedure for ANSYS FLUENT work follows the above components. For the present work, in this case to ensure that 2D, later this mesh file is imported into ANSYS FLUENT for analysis. In the FLUENT, the analysis will be carried out. The postprocessing is carried according to the requirements.

\section{CFD Analysis}

3.1. Aerodynamic Analysis of Wortmann FX60-100 2D Airfoil. The physical characteristics of selected airfoil such as thickness 0.09996 , camber 0.03503 , and area 0.05972 . The purpose of choosing this specific airfoil is low Reynolds number (Re) which gives better aerodynamic characteristic performance as compared to the other airfoils and it is the one of the best airfoils which is used in design of micro aerial vehicle [4].

3.2. Computational Domain Construction. In this study only the $2 \mathrm{D}$ airfoil is considered. A schematic geometry of the wing is shown in Figure 5, which is constructed and extracted from ICEM CFD.

The physical problem under consideration is the flow over an airfoil in a computational domain as shown in Figure 6.

The basic requirement of the airfoil location is to make the outer boundaries sufficiently far away from the airfoil so that they do not have much impact on the computed results; the relative position of the airfoil is shown in Figure 6.

3.3. Meshing. The computational model is created in ICEMCFD as per the dimensions shown in Figure 7. Later the structure grid is generated.

The grid distribution around the wing is shown in Figure 7. A 2D hexahedral grid having approximately total 13232 elements has been generated using ICEM-CFD. The

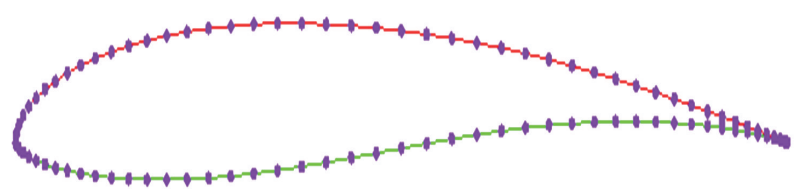

FIGURE 5: Geometry details of 2D airfoil.

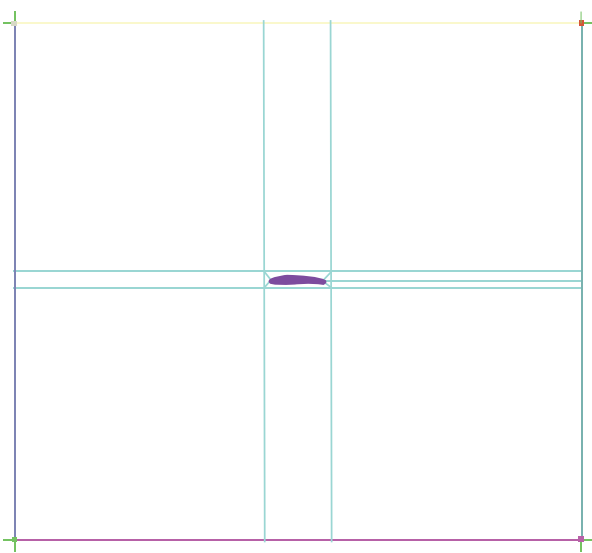

Figure 6: Computational domain.

quality of grid has been maintained as Min $=0.540005$, $\max$ $=0.99995$ in terms of skewness, which can be defined by

$$
Q_{\mathrm{EVS}}=\left[S_{\mathrm{EQ}}-\frac{S}{S_{\mathrm{EQ}}}\right] \text {, }
$$

where $S$ is volume (3D) of the mesh element and $S_{\mathrm{EQ}}$ is the maximum area (2D) or volume (3D) of an equilateral cell the circumscribing radius of which is identical to the mesh element by definition $0 \leq Q_{\mathrm{EVS}} \leq 1$.

3.4. FLUENT Setup for 2D Airfoil. The FLUENT setup for 2D airfoil includes solvers settings, operating conditions, boundary conditions, and reference values. In this work the pressure, density, and temperature are taken to be constant for sea level conditions because of laminar flow. The velocity taken $11.5 \mathrm{~m} / \mathrm{s}$ is maximum velocity of the designed micro aerial vehicle [4].

\subsection{Test Cases}

Case 1: Solved by Ansys Fluent. FLUENT is a state-of-theart computer program for modeling the fluid flow and heat transfer in complex geometries. It provides comprehensive modeling capabilities for a wide range of incompressible and compressible, laminar and turbulent fluid flow problems. FLUENT uses unstructured meshes in order to reduce the amount of time spent on grid generation simplify the geometry modeling. In this work the parameters are considered for sea level standard conditions and the velocity considered as 


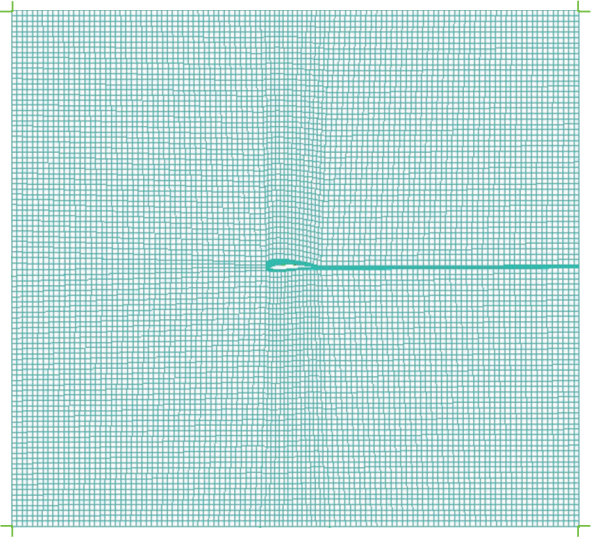

FIgURE 7: Grid distributions around the 2D airfoil.

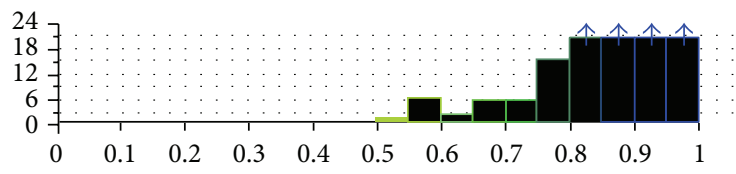

Figure 8: Histogram of angle.

$11.5 \mathrm{~m} / \mathrm{s}$ for this case changes the AOA from $-10^{\circ}$ to $+15^{\circ}$ for every $5^{\circ}$ of intervals (Figure 8 ).

Case 2: Solved by Xfoil. The process of obtaining the aerodynamic characteristics for 2D airfoil was fairly straightforward and done using a freely available subsonic airfoil development interactive program called Xfoil. In this program airfoil coordinates are entered to get the geometry. Later entered the Reynolds number and AOA sequence from $-10^{\circ}$ to $+15^{\circ}$, and then the aerodynamic characteristics have been generated such as lift, drag, moment coefficient, and pressure coefficient:

$$
\operatorname{Re}=\frac{\rho V C}{\mu},
$$

where $\rho=1.225 \mathrm{~kg} / \mathrm{m}^{3}, V=11.5 \mathrm{~m} / \mathrm{s}, \operatorname{chord}(C)=1 \mathrm{~m}, \mu=$ $1.7894 * 10^{-5}$, and

$$
\operatorname{Re}=0.086 * 10^{6} .
$$

\section{Results and Discussion}

4.1. Variation of Nondimensional Coefficients with AOA in FLUENT. In this section, the force coefficients are computed for the wing for different $\mathrm{AOA}$ at $11.5 \mathrm{~m} / \mathrm{s}$ speed using fluent software. Figure 9 shows the variation of the nondimensional $C_{l}$ versus AOA for all the test cases.

At an AOA, $\alpha=5^{\circ}$, the reported value of it is $C_{l}=0.9$ caused by the low pressure region on the upper surface. As the $\mathrm{AOA}$ is increased, the low pressure region on the upper wing surface increases. The coefficient of lift is almost linear with the AOA up to $10^{\circ}$. Boundary layer separation occurs beyond AOA $10^{\circ}$, which introduces unsteadiness to the aerodynamic performance. At $\mathrm{AOA}$ of $10^{\circ}$, the flow on the upper surface

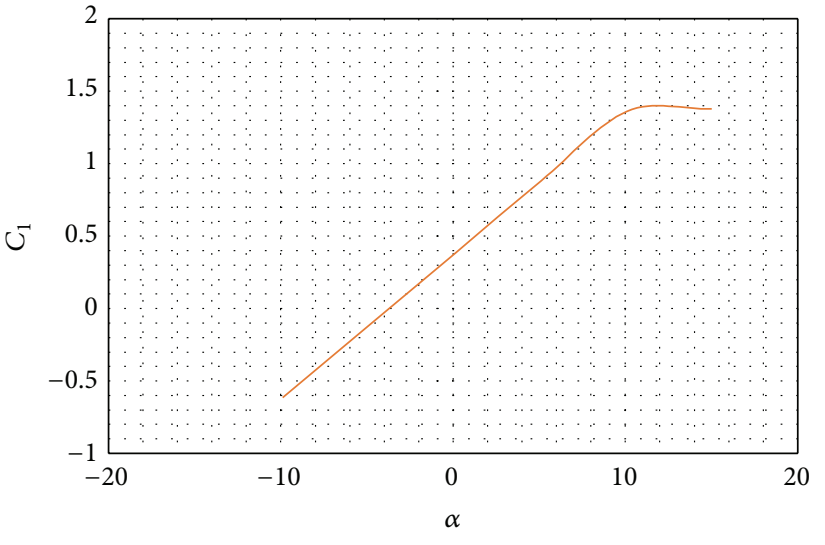

_ Present data

FIGURE 9: Lift coefficients versus AOA.

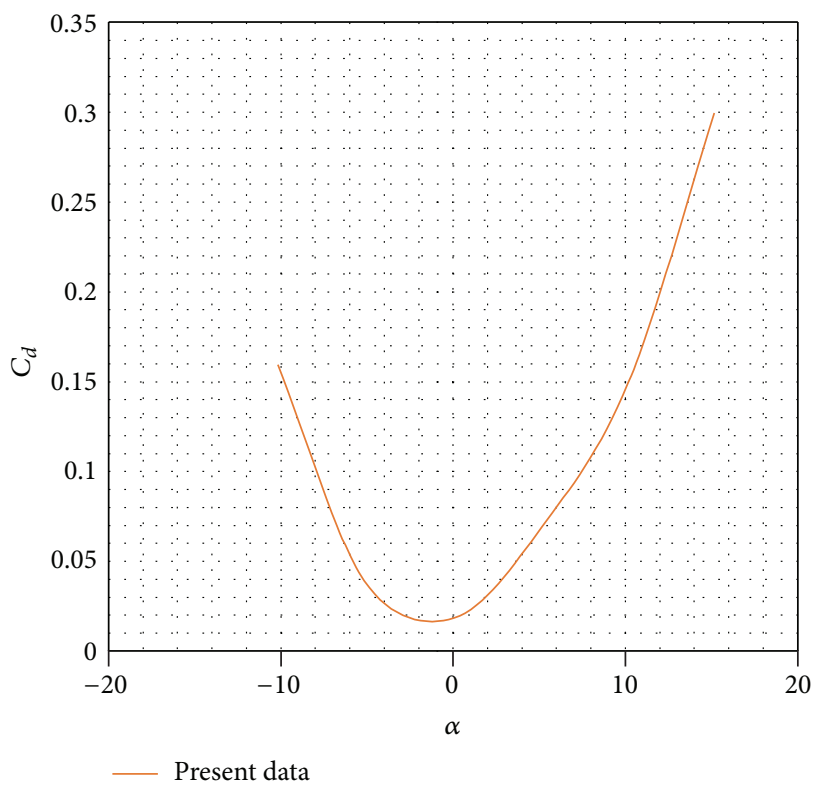

Figure 10: Drag coefficients versus AOA.

starts to get separated from a large portion of the surface; however, the lift forces sometimes may increase with the AOA. The reason might be tip vortices suction near tip area which would result in additional lift or the flow from the high pressure region on the lower wing surface tries to reach the low pressure region on the upper wing surface.

Figure 10 shows the variation of the nondimensional $C_{d}$ versus $\alpha$ for all the test cases.

As $\mathrm{AOA}$ is increased beyond $15^{\circ}$, massive separation occurs on most of the upper surface, the lift decreases, and drag starts to increases as seen in the Figure 10; it also indicates constant increase in the drag from $5^{\circ}$ until it reaches its maximum value at $15^{\circ}$. From the results, at $\alpha=10^{\circ}$, the 


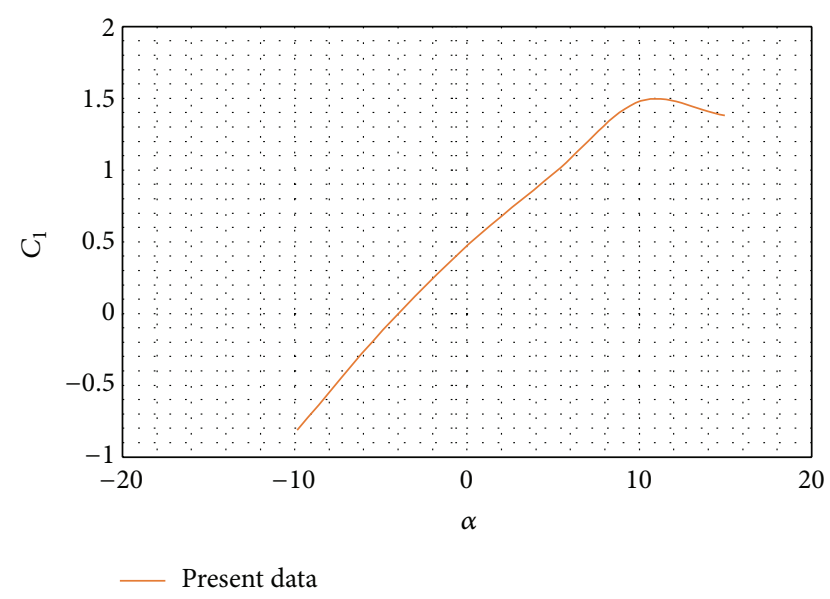

FIgURE 11: Lift coefficients versus AOA.

values of $C_{l}$ and $C_{d}$ are 1.38 and 0.15 , respectively, and their ratio is 9.2 .

Flow Conditions. $P_{\infty}=1.01325 * 10^{5} \mathrm{~N} / \mathrm{m}^{2}, \rho_{\infty}=1.225 \mathrm{Kg} / \mathrm{m}^{3}$, $\mu_{\infty}=1.789 * 10^{-5} \mathrm{Kg} / \mathrm{m} \mathrm{s}, \operatorname{Re}=0.087 * 10^{6}, V=11.5 \mathrm{~m} / \mathrm{s}$, and $\alpha=-10^{\circ}$ to $15^{\circ}$.

4.2. Variation of Nondimensional Coefficients with $A O A$ in $X$ foil. In this section, the force coefficients are computed for the wing for different AOA at $11.5 \mathrm{~m} / \mathrm{s}$ speed using Xfoil panel code method. Figure 11 shows the variation of the nondimensional $C_{l}$ versus AOA for all the test cases.

At an AOA, $\alpha=5^{\circ}$, the reported value of it is $C_{l}=1$ caused by the low pressure region on the upper surface. As the $\mathrm{AOA}$ is increased, the low pressure region on the upper wing surface increases. The coefficient of lift is almost linear with the AOA up to $10^{\circ}$. Boundary layer separation occurs beyond $\mathrm{AOA} 10^{\circ}$, which introduces unsteadiness to the aerodynamic performance.

At $\mathrm{AOA}$ of $10^{\circ}$, the flow on the upper surface starts to get separated from a large portion of the surface; however, the lift forces sometimes may increase with the AOA. The reason might be tip vortices suction near tip area which would result in additional lift or the flow from the high pressure region on the lower wing surface tries to reach the low pressure region on the upper wing surface.

Figure 12 shows the variation of the nondimensional $C_{d}$ versus AOA for all the test cases.

As $\mathrm{AOA}$ is increased beyond $15^{\circ}$, massive separation occurs on most of the upper surface, the lift decreases, and drag starts to increase as seen in the Figure 12. It also indicates constant increase in the drag from $5^{\circ}$ until it reaches its maximum value at $15^{\circ}$. From the results, at $\alpha=10^{\circ}$, the values of $C_{l}$ and $C_{d}$ are 1.38 and 0.15 , respectively, and their ratio is 9.2.

Flow Conditions. $P_{\infty}=1.01325 * 10^{5} \mathrm{~N} / \mathrm{m}^{2}, \rho_{\infty}=1.225 \mathrm{Kg} / \mathrm{m}^{3}$, $\mu_{\infty}=1.789 * 10^{-5} \mathrm{Kg} / \mathrm{m} \mathrm{s}, \mathrm{Re}=0.087 * 10^{6}, V=11.5 \mathrm{~m} / \mathrm{s}$, and $\alpha=-10^{\circ}$ to $15^{\circ}$.

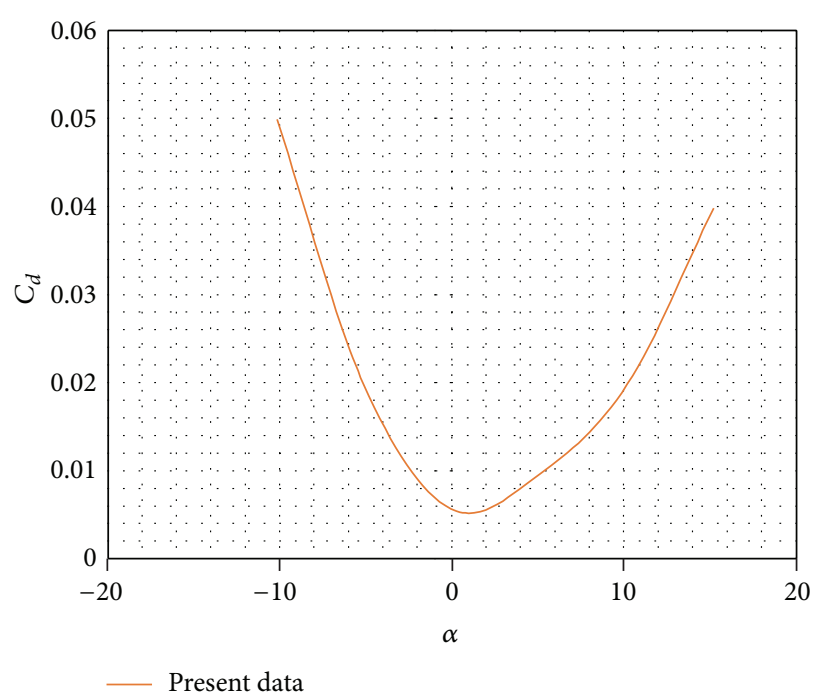

Figure 12: Drag coefficients versus AOA.

4.3. Contours of Pressure Coefficient. Figures 13(a)-13(f) show $2 \mathrm{D}$ contour of pressure coefficient plotted for various AOA conditions, that is, $-10^{\circ}$ through $+15^{\circ}$ of AOA. The dark red color contour indicates the maximum pressure location over the wing geometry, whereas dark blue contour indicates minimum pressure location.

\section{Validation of Wortmann FX60-100 2D Airfoil}

Figures 14 to 16 give the overall view of the study carried out in this work for 2D case; two cases are considered under this with 6 angles of attack $(\alpha)$, that is, $-10^{\circ}$ through $+15^{\circ}$ with interval of $5^{\circ}$ of AOA.

Flow Conditions. $P_{\infty}=1.01325 * 10^{5} \mathrm{~N} / \mathrm{m}^{2}, \rho_{\infty}=1.225 \mathrm{Kg} / \mathrm{m}^{3}$, $\mu_{\infty}=1.789 * 10^{-5} \mathrm{Kg} / \mathrm{m} \mathrm{s}, \mathrm{Re}=0.087 * 10^{6}, V=11.5 \mathrm{~m} / \mathrm{s}$, and $\alpha=-10^{\circ}$ to $15^{\circ}$.

Figure 14 shows the plot of the coefficient of lift versus angle of attack for the FX60100 2D airfoil and the plot shows very good correlation between the two different solvers results as well as the experimental data obtained from the literature-1. The plot shows that the results obtained from the two solvers were with the maximum variation of $C_{l}$ as $0 \%$. The above obtained solution difference as compared to the experimental data was with maximum variation of $C_{l}$ as $10 \%$. Maximum $C_{l}$ for the above reference values occurs at AOA of $15^{\circ}$ and for the present work, maximum $C_{l}$ occurs at AOA of $15^{\circ}$. On comparing this, it can be found that there is close agreement in the behavior of the wing. So it can be observed that the present results are close to [4].

Figure 15 shows the data obtained for the coefficient of drag for the FX60100 2D airfoil and the plot shows very good correlation between the two different solvers results as well as the experimental data obtained from the literature-1, but slightly higher than that obtained percentage variation in the drag by other, the drag predicted by the fluent, Xfoil, and the 


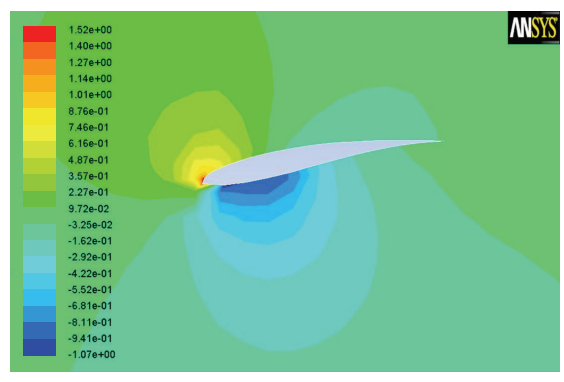

(a) $-10^{\circ} \mathrm{AOA}$

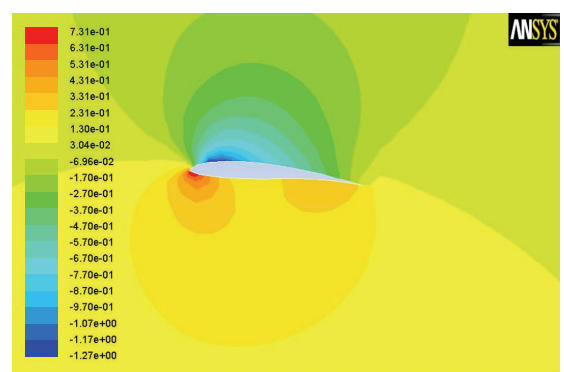

(d) $5^{\circ} \mathrm{AOA}$

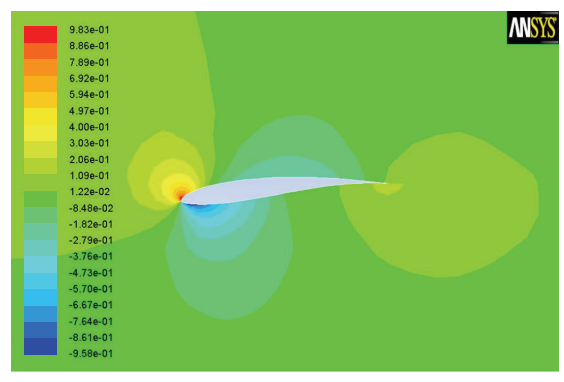

(b) $-5^{\circ} \mathrm{AOA}$

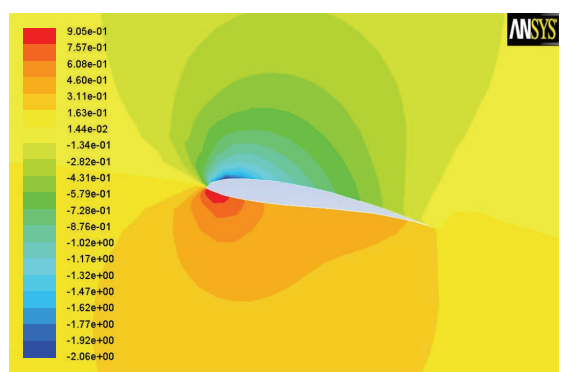

(e) $10^{\circ} \mathrm{AOA}$

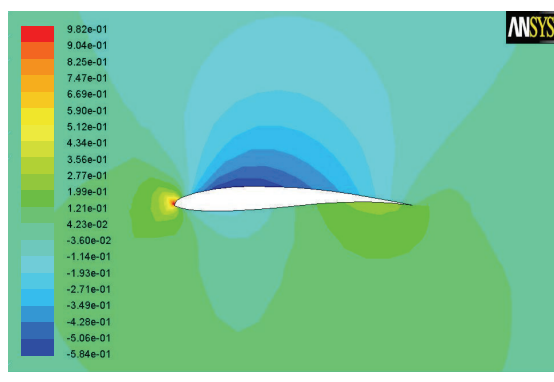

(c) $0^{\circ} \mathrm{AOA}$

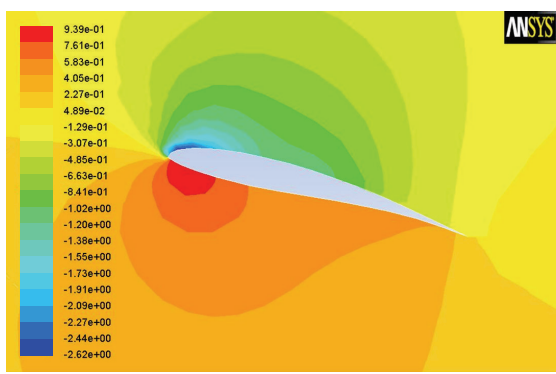

(f) $15^{\circ} \mathrm{AOA}$

Figure 13: Contours of pressure efficient $-10^{\circ}$ through $+15^{\circ}$ of AOA.

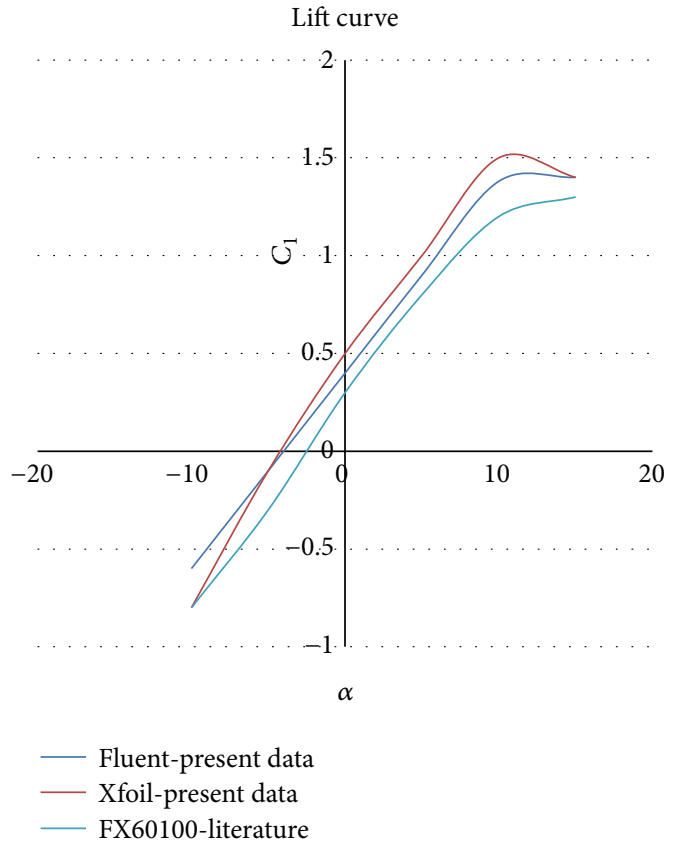

FigUre 14: Lift coefficients versus AOA.

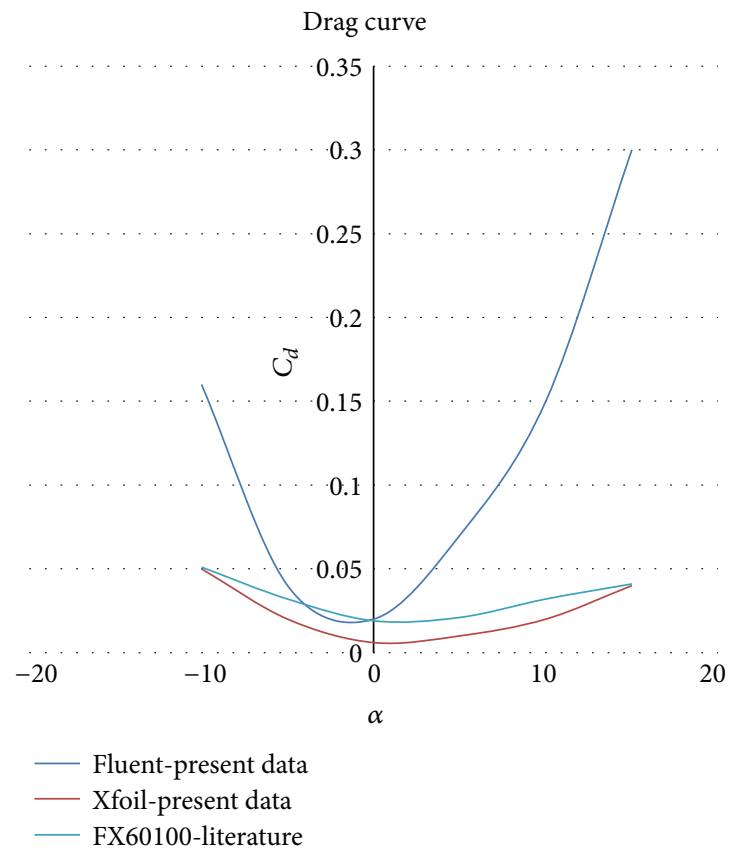

Figure 15: Drag coefficients versus AOA. experimental values. The solution difference as compared to the experimental data varied from $10.9 \%$ to $25.9 \%$.

Figure 16 shows the plot of $C_{l}$ versus $C_{d}$ for the FX60100 $2 \mathrm{D}$ airfoil. The plot shows that the in results obtained from the two solvers the $C_{l}$ maximum varied $0 \%$. The solution difference as compared to the experimental data the $C_{l}$ maximum varied $10 \%$. But slightly higher than that obtained percentage variation in the drag by other, the drag predicted by the fluent, Xfoil, and the experimental values. The solution difference as compared to the experimental data varied from $10.9 \%$ to $25.9 \%$.

\section{Conclusions from the 2D Airfoil Study in CFD}

The following conclusions can be made from the $2 \mathrm{D}$ airfoil study. 


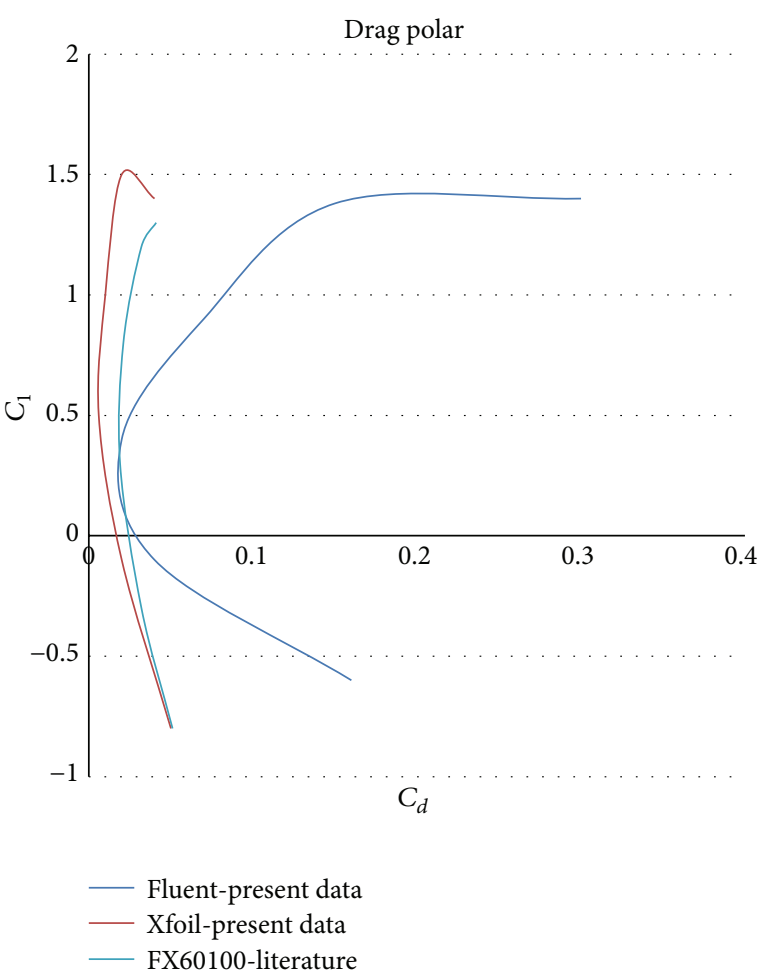

Figure 16: Lift coefficients versus drag coefficients.

(i) It is observed from the Figures 13(a)-13(f) are that of the contours of pressure coefficient on the airfoil and they depict the flow separation by the start of the strand on the surface of the airfoil. It is seen that at higher angles of attack the separation is much more prominent and closer to the leading edge of the airfoil.

(ii) The coefficient of lift versus angle of attack for the FX60100 2D airfoil and the plot show very good correlation between the two different solvers results as well as the experimental data obtained from the literature-1.

(iii) The coefficient of drag is high in FLUENT results as compared to the Xfoil and experimental data obtained from the literature-1. The exact drag prediction is highly difficult.

(iv) The study of the 2D airfoil Wortmann FX 60100 shows a good agreement between the results of CFD analysis with the reference values [4].

(v) A major reason for the CFD coefficient of moment analysis was not to study but to verify the data obtained from the wind tunnel tests. Also none of the airfoils and models tested had a coefficient of moment data.

\section{Conflict of Interests}

The author declares that there is no conflict of interests regarding the publication of this paper.

\section{References}

[1] S. P. Sane, "The aerodynamics of insect flight," The Journal of Experimental Biology, vol. 206, pp. 4191-4208, 2003.

[2] M. H. Dickinson and K. G. Gotz, "Unsteady aerodynamic performance of model wings at low Reynolds numbers," The Journal of Experimental Biology, vol. 174, pp. 45-64, 1993.

[3] J.-M. Moschetta, "A Fixed-Wing Micro Air Vehicle with Hovering Capability," Department of Aerodynamics, Paris, France, 2010.

[4] F. Barnhart, M. Cuipa, D. Stefanik, and Z. Swick, Micro-Aerial Vehicle Design with Low Reynolds Number Airfoils, 2004. 

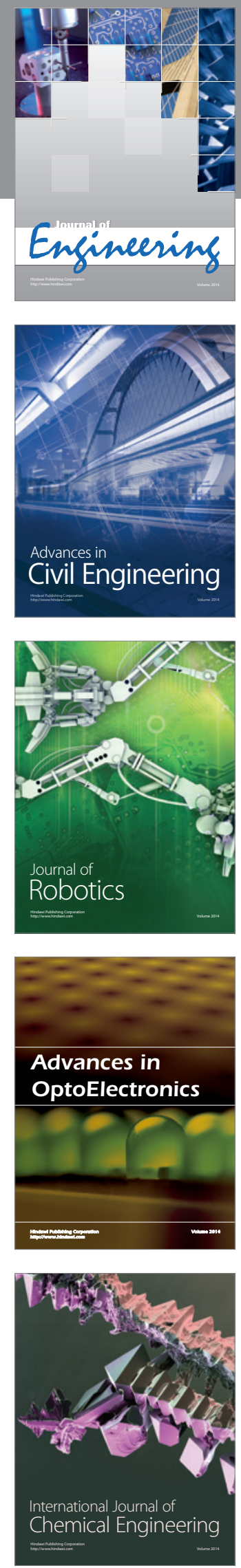

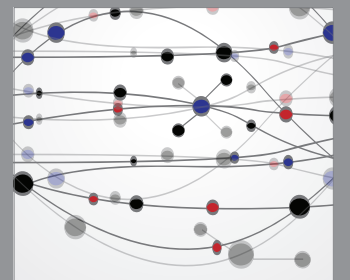

The Scientific World Journal
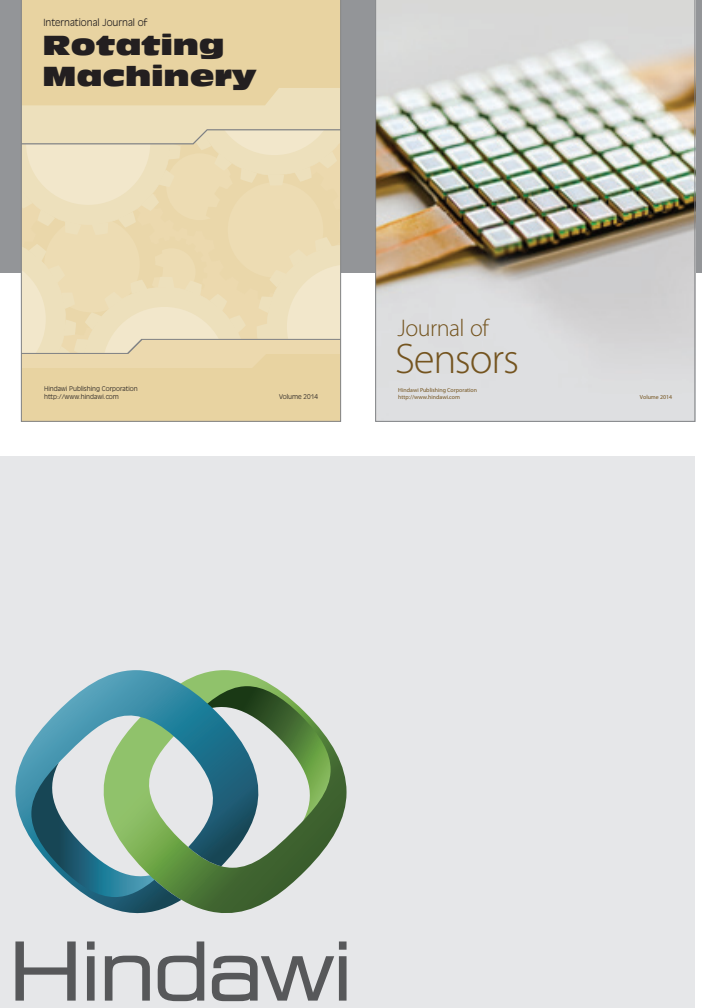

Submit your manuscripts at http://www.hindawi.com
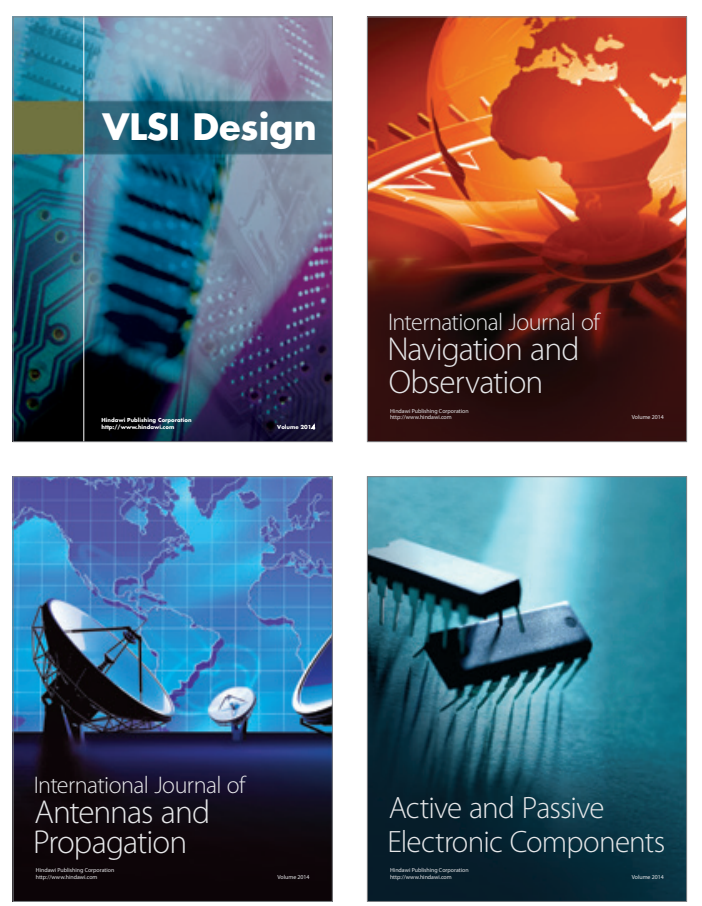
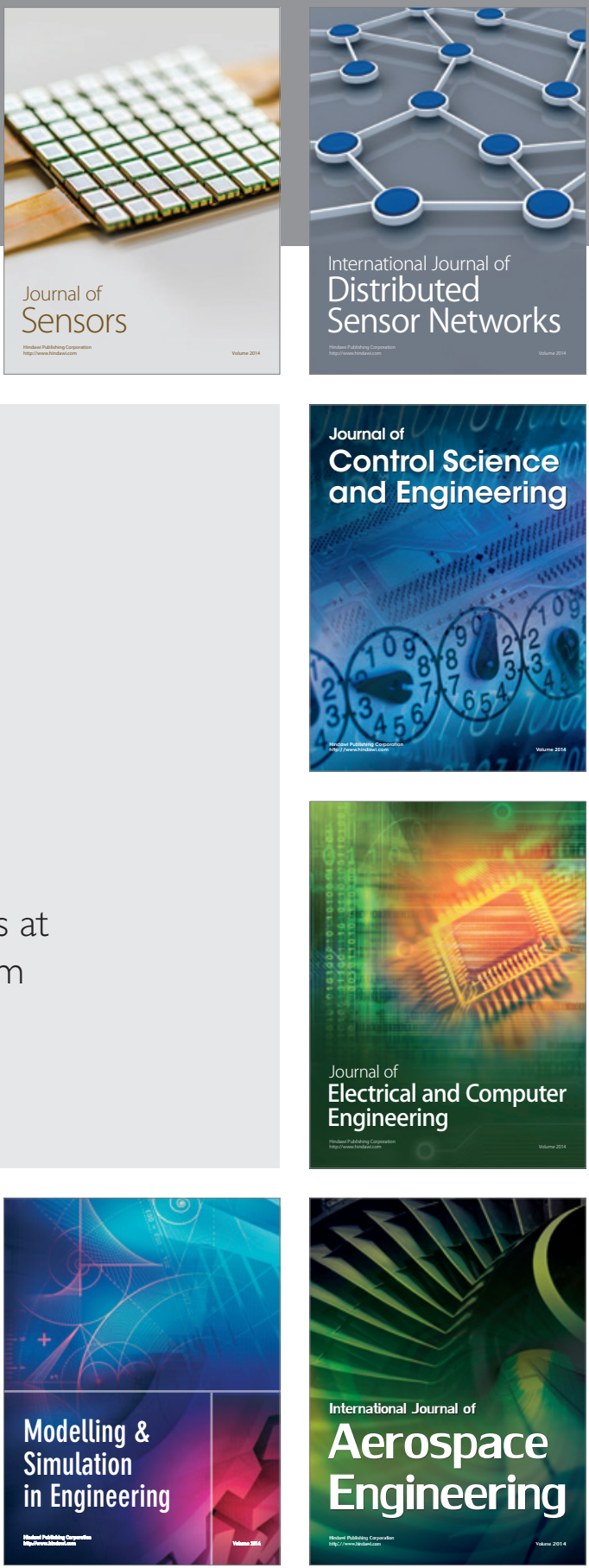

Journal of

Control Science

and Engineering
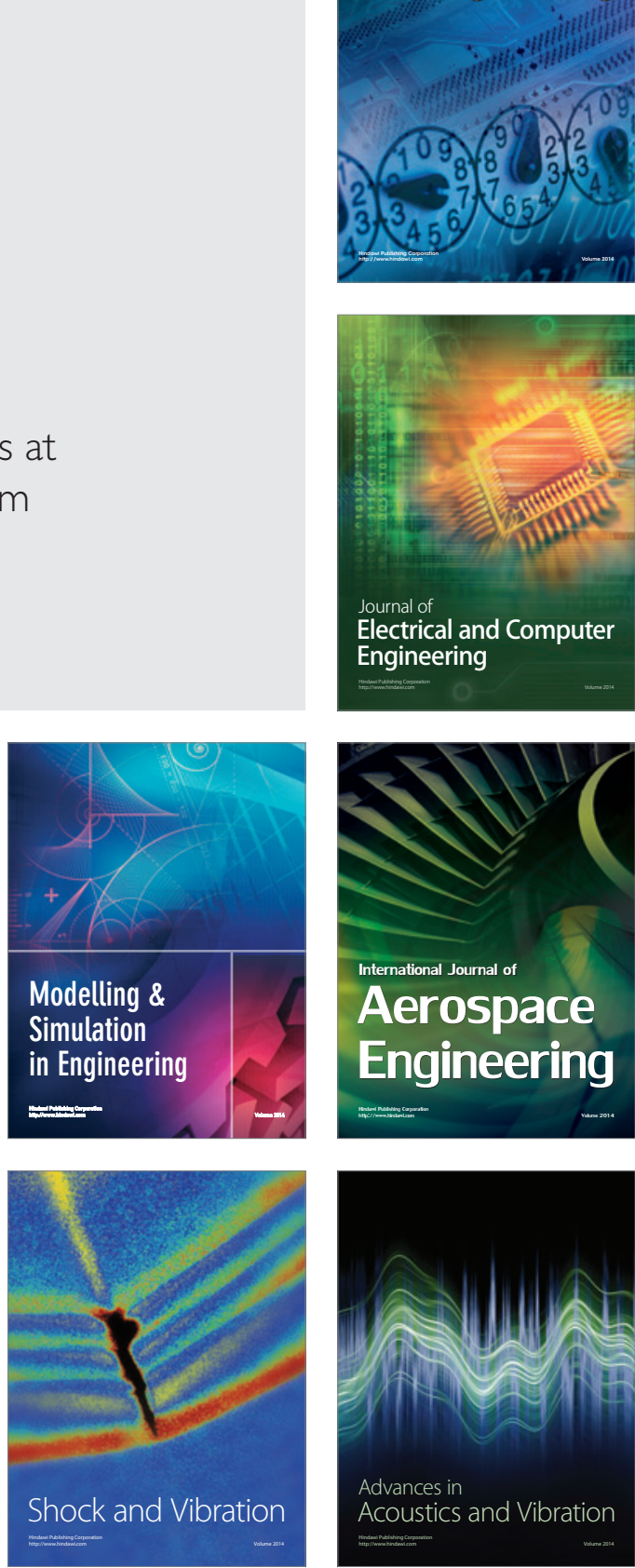